International Journal of Pure and Applied Mathematics

Volume 93 No. 2 2014, 201-215

ISSN: 1311-8080 (printed version); ISSN: 1314-3395 (on-line version)

url: http://www.ijpam.eu

doi: http://dx.doi.org/10.12732/ijpam.v93i2.5

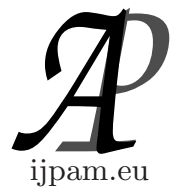

\title{
BLOCK METHOD FOR NUMERICAL SOLUTION OF DIFFERENTIAL-ALGEBRAIC EQUATIONS WITH HESSENBERG INDEX-3
}

\author{
O.A. Akinfenwa ${ }^{1}$, S.A. Okunuga ${ }^{2}$ \\ 1,2 Department of Mathematcs \\ University of Lagos \\ Akoka, Lagos, NIGERIA
}

\begin{abstract}
A Block Second Derivative Formula (BSDF) of order seven for the numerical solution of Hessenberg Differential Algebraic Equations (DAEs) of index 3 is presented. This is achieved by constructing a Continuous Second Derivative Formula (CSDF) used to obtain the main and additional methods which are combined to form a single block of methods that simultaneously provide the approximate solutions for the Hessenberg DAEs of index 3. The error constant and stability properties of the BSDF are discussed. The performance of the method is demonstrated on some numerical examples to show the accuracy and efficiency of the method.
\end{abstract}

AMS Subject Classification: 65L05, 65L06, 65L08

Key Words: differential-algebraic equations, block second derivative formula, stability analysis

\section{Introduction}

Many important mathematical problems can be modeled as systems of Differential-Algebraic Equations (DAEs). These problems have a wide range of applications in various branches of science and engineering. These include mechanical or multibody systems, chemical processes, optimal control, electric circuit design, analytical surveys and dynamical systems. In the literature,

Received: December 30, 2013

(c) 2014 Academic Publications, Ltd.

$\S$ Correspondence author url: www.acadpubl.eu 
some numerical methods have been developed for the solution of DAEs such as the BDF (see [2], [3], [9]), implicit Runge-Kutta methods [3], Pade and Chebysev approximation methods (see [4] [5] [6]) and variational iterative method [12]. These methods are only directly suitable for low-index problems and often require that the problem, have special structure. Although many important applications can be solved by these methods, there is a need for more efficient, accurate and reliable techniques.

A system of ordinary differential equations (ODEs) with algebraic constraints which can be written in the general form as

$$
F\left(t, y, y^{\prime}\right)=0
$$

is called differential algebraic equation, where $\frac{\partial F}{\partial y^{\prime}}$ may be singular and the structure and rank of the of this Jacobian matrix may depend on the solution $y(t)$. An important special case of (1) is the semi-explicit DAE of the form

$$
\left.\begin{array}{ll}
y^{\prime}=f_{1}(y(t), z(t)), & y\left(t_{0}\right)=y_{0} \\
f_{2}(y(t), z(t))=0, & z\left(t_{0}\right)=z_{0}
\end{array}\right\}
$$

whose index is 1 if $\frac{\partial g}{\partial z}$ is nonsingular, since in principle differentiating $f_{2}(y(t)$, $z(t))=0$, once yields $z^{\prime}$. Thus the distinction between the differential variables $y(t)$ and algebraic variables $z(t)$. The algebraic variables may be less smooth than the differential variables by one derivative. In the general case, each component of y may contain a mix of differential and algebraic components, which makes the numerical solutions of such high-index problems harder and with higher risk.

Most of the higher-index problems encountered in practice can be expressed as a combination of more restrictive structures of ODEs coupled with constraints. In such systems the algebraic and differential variables are explicitly identified and all the algebraic variables may be eliminated using the same number of differentiations. These type of DAEs are called Hessenberg DAEs.

Definition 1.1 (Hessenberg Index-1)

$$
\left.\begin{array}{l}
y^{\prime}=f_{1}(t, y, z) \\
f_{2}(t, y, z)=0
\end{array}\right\}
$$

Here the Jacobian matrix function $f_{2 z}$ is assumed to be nonsingular for all t. This is also often referred to as a semi-explicit index- 1 system.

Definition 1.2 (Hessenberg Index-2)

$$
\left.\begin{array}{l}
y^{\prime}=f_{1}(t, y, z) \\
f_{2}(t, y)=0
\end{array}\right\}
$$


the variables $\mathrm{z}$ of the algebraic part is absent from the constraint and the product of Jacobians $f_{2 y} f_{1 z}$ is nonsingular for all $t$. Thus, all algebraic variables play the role of index-2 therefore this is a pure index-2 DAE.

Definition 1.3 (Hessenberg Index-3)

$$
\begin{aligned}
& y^{\prime}=f_{1}(t, y, w, z), \\
& z^{\prime}=f_{2}(t, y, w), \\
& f_{3}(t, w)=0
\end{aligned}
$$

In this case the product of three matrix functions $f_{3 w} f_{2 y} f_{1 z}$ is nonsingular. The index of a Hessenberg DAE is found, as in the general case, by differentiation. However, only the algebraic constraints must be differentiated.

The aim in this paper is to develop a Block Second Derivative formula (BSDF) of order $\mathrm{k}+2$ for the solution of Hessenberg DAE of the form (5) that will be efficient, reliable and accurate. Block methods were first introduced by Milne [14] for use only as a means of obtaining starting values for predictorcorrector algorithms and has since then been developed by several researchers (see [[8],[16],[17],[18]). Recently, Akinfenwa and Okunuga [1] and Naghmeh et.al.[15] developed block method for the solution of semi explicit index 1 DAEs This paper as in [1] presents a block method which preserves the Runge-Kutta traditional advantage of being self-starting and efficient, since it requires $\mathrm{m}$ function evaluations per integration step, where $\mathrm{m}$ is the number of functions in the block method.

\section{Construction of BSDF}

In this section, we construct the continuous scheme of the main and additional methods known as the second derivative formula (SDF) and are combined to form the BSDF on the interval from $t_{n}$ to $t_{n+k}=t_{n}+k h$ where $h$ is the chosen step-length and $k$ is the step number of the method. We assume that the exact solution $y(t)$ on the interval $\left[t_{n}, t_{n+k}\right]$ is locally represented by $Y(t)$ given by

$$
Y(t)=\sum_{j=0}^{s+r-1} l_{j} \varphi_{j}(t),
$$

$l_{j}$ are unknown coefficients to be determined, and $\varphi_{j}(t)$ are polynomial basis function of degree $s+r-1$ such that the number of interpolation points $s$ and the number of distinct collocation points $r$ are respectively chosen to satisfy $1 \leq s<k, k=5$, and $r>0$. The proposed class of methods is thus constructed 
by imposing the following conditions

$$
\begin{gathered}
\sum_{j=0}^{k+2} l_{j} t_{n+i}^{j}=y_{n+i}, \quad i=0, \\
\sum_{j=0}^{k+2} l_{j} j t_{n+i}^{j}-1=f_{n+i}, \quad i=0, \ldots, k, \\
\sum_{j=0}^{k+2} l_{j} j(j-1) t_{n+i}^{j}-2=g_{n+i}, \quad i=k,
\end{gathered}
$$

assuming that $y_{n+i}=Y\left(t_{n}+i h\right)$, denote the numerical approximation to the exact solution $\left.y\left(t_{n+i}\right) f_{n+i}=Y^{\prime}\left(t_{n}+i h\right), y_{n+j}\right)$, denote the approximation to $y^{\prime}\left(t_{n+i}\right) n$ is the grid index. It should be noted that equation $(7),(8)$ and (9) lead to a system of $k+3$ equations which must be solved to obtain the coefficients $l_{j}, j=0, \ldots, 7$. The continuous second derivative formula (CSDF) is then obtained by substituting the values of $l_{j}$ into equation (6). After some algebraic reduction, the CSDF yields the expression in the form (10)

$$
\left.Y(t)=\alpha_{j}(t) y_{n}+h \sum_{j=0}^{5} \beta_{j}(t) f_{n+j}+h^{2} \gamma_{5}(t) g_{n+5}\right)
$$

where $\alpha_{j}(t), j=0, \beta_{j}(t), j=0,1, \ldots, 5$ and $\gamma_{5}(t)$ are continuous coefficients. The CSDF (10) is then used to generate the main and additional discrete formulas by evaluating at points $t=t_{n+5}$ and $\left.t=t_{n+1}, \ldots, t_{n+4}\right]$. These discrete formulas given in (11) and (12) are then combined and implemented simultaneously as a single block formula for the solution of the Hessenberg index 3 DAEs.

$$
\begin{aligned}
y_{n+5}=y_{n}+\frac{305 h}{1008} f_{n}+\frac{11875 h}{8064} f_{n+1}+\frac{625 h}{1512} f_{n+2}+\frac{3125 h}{2016} f_{n+3}+\frac{625 h}{1008} f_{n+4} \\
+ \\
+\frac{15515 h}{24192} f_{n+5}-\frac{275 h^{2}}{2016} g_{n+5},
\end{aligned}
$$




$$
\left.\begin{array}{rl}
y_{n+1}= & y_{n}+\frac{2627 h}{8400} f_{n}+\frac{4919 h}{4480} f_{n+1}-\frac{6347 h}{7560} f_{n+2}+\frac{2563 h}{3360} f_{n+3}-\frac{307 h}{560} f_{n+4} \\
& +\frac{129571 h}{604800} f_{n+5}+\frac{863 h^{2}}{197} g_{n+5} \\
y_{n+2}= & y_{n}+\frac{943 h}{3150} f_{n}+\frac{3797 h}{2520} f_{n+1}-\frac{38 h}{945} f_{n+2}+\frac{283 h}{630} f_{n+3}-\frac{227 h}{630} f_{n+4} \\
& +\frac{5489 h}{37800} f_{n+5}-\frac{37 h^{2}}{630} g_{n+5} \\
y_{n+3}= & y_{n}+\frac{849 h}{2800} f_{n}+\frac{6567 h}{4480} f_{n+1}+\frac{127 h}{280} f_{n+2}+\frac{1233 h}{1120} f_{n+3}-\frac{291 h}{560} f_{n+4} \\
& +\frac{4393 h}{22400} f_{n+5}-\frac{87 h^{2}}{1120} g_{n+5} \\
y_{n+4}= & y_{n}+\frac{158 h}{525} f_{n}-\frac{52 h}{35} f_{n+1}+\frac{344 h}{945} f_{n+2}+\frac{176 h}{105} f_{n+3}+\frac{2 h}{35} f_{n+4} \\
& -\frac{548 h}{4725} f_{n+5}-\frac{16 h^{2}}{315} g_{n+5} .
\end{array}\right\}
$$

\section{Analysis of the BSDF}

The SDFs can be represented by a matrix finite difference equation in block form as

$$
A^{(1)} Y_{\omega}=A^{(0)} Y_{\omega-1}+h B^{(1)} F_{\omega}+h B^{(0)} F_{\omega-1}+h^{2} C^{1} G_{\omega},
$$

where:

$$
\begin{aligned}
& Y_{\omega}=\left(y_{n+1}, y_{n+2}, \ldots, y_{n+5},\right)^{T}, \\
& Y_{\omega-1}=\left(y_{n-4}, y_{n-3}, \ldots, y_{n}\right)^{T}, \\
& F_{\omega}=\left(f_{n+1}, f_{n+2}, f_{n+3}, \ldots, f_{n+5}\right)^{T}, \\
& F_{\omega-1}=\left(f_{n-4}, f_{n-3}, \ldots, f_{n}\right)^{T}, \\
& G_{\omega}=\left(g_{n+1}, g_{n+2}, \ldots, g_{n+5}\right)^{T},
\end{aligned}
$$

for $\omega=1, \ldots \pi_{N}, \pi_{N}$ is number of partition on [a,b] and $n=0,5, \ldots, N-5$.

And $A^{(1)}$ is an identity matrix of dimension 5 , while the matrices $A^{(0)}, B^{(1)}$, $B^{(0)}$ and $C^{(1)}$ are 5 by 5 matrices whose entries are given by the combined 
coefficients of the methods obtained in (10) given as follows:

$$
\begin{gathered}
A^{(0)}=\left(\begin{array}{ccccc}
0 & 0 & 0 & 0 & 1 \\
0 & 0 & 0 & 0 & 1 \\
0 & 0 & 0 & 0 & 1 \\
0 & 0 & 0 & 0 & 1 \\
0 & 0 & 0 & 0 & 1
\end{array}\right) \\
B^{(1)}=\left(\begin{array}{ccccc}
\frac{4919}{4480} & -\frac{6347}{7560} & \frac{2563}{3360} & -\frac{307}{560} & \frac{129571}{604800} \\
\frac{3797}{2520} & -\frac{38}{945} & \frac{283}{630} & -\frac{227}{630} & \frac{5589}{37800} \\
\frac{6576}{4480} & \frac{127}{280} & \frac{1233}{1120} & -\frac{291}{560} & \frac{4393}{22400} \\
\frac{52}{35} & \frac{344}{945} & \frac{176}{105} & \frac{2}{35} & \frac{548}{4725} \\
\frac{11875}{8064} & \frac{625}{1512} & \frac{3125}{2016} & \frac{625}{1008} & \frac{15515}{24192}
\end{array}\right) \\
B^{(0)}=\left(\begin{array}{ccccc}
0 & 0 & 0 & 0 & \frac{2627}{8400} \\
0 & 0 & 0 & 0 & \frac{943}{3150} \\
0 & 0 & 0 & 0 & \frac{849}{2800} \\
0 & 0 & 0 & 0 & \frac{158}{525} \\
0 & 0 & 0 & 0 & \frac{305}{2016}
\end{array}\right) \\
C^{(1)}=\left(\begin{array}{ccccc}
0 & 0 & 0 & 0 & -\frac{863}{10080} \\
0 & 0 & 0 & 0 & -\frac{37}{630} \\
0 & 0 & 0 & 0 & -\frac{87}{1120} \\
0 & 0 & 0 & 0 & -\frac{16}{315} \\
0 & 0 & 0 & 0 & -\frac{275}{2016}
\end{array}\right)
\end{gathered}
$$

Following Fatunla [7] and Lambert [13], the local truncation error associated with each of the method in the BSDF can be defined by a linear difference operator

$$
L[y(t) ; h]=\sum_{j=0}^{k} \alpha_{j} y_{n+j}-h \sum_{j=0}^{k} \beta_{j} y_{n+j}^{\prime}+h^{2} \gamma_{k} y_{n+k}^{\prime \prime} .
$$

Assuming that $y(t)$ is sufficiently differentiable, we can write the terms in (14) as a Taylor series about the point $t$ to obtain the expression

$$
L[y(t) ; h]=C_{0} y(t)+C_{1} h y^{\prime}(t)+C_{2} h^{2} y^{\prime \prime}(t)+\ldots,+C_{q} h^{q} y^{q}(t)+\ldots,
$$

where the constant coefficients $C_{p}, p=0,1,2, \ldots$, are given as follows:

$$
\begin{aligned}
& C_{0}=\sum_{j=0}^{k} \alpha_{j}, \\
& C_{1}=\sum_{j=1}^{k} j \alpha_{j}-\beta_{j}, \\
& C_{2}=\frac{1}{2 !}\left(\sum_{j=1}^{k} j^{2} \alpha_{j}-2 j \beta_{j}-2 \gamma_{k}\right),
\end{aligned}
$$


$C_{p}=\frac{1}{p !}\left(\sum_{j=1}^{k-1} j^{p} \alpha_{j}-p(j)^{p-1} \beta_{j}-p(p-1) k^{p-2} \gamma_{k}\right)$.

According to Henrici [11], we say that the methods in (11) have a maximal order of accuracy $\mathrm{p}$ if

$$
L[y(t) ; h]=\bigcirc\left(h^{m+1}\right), \quad C_{0}=C_{1}=\ldots=C_{p}=0, C_{p+1} \neq 0 .
$$

Therefore, $C_{p+1}$ is the error constant and $C_{p+1} h^{p+1} y^{(p+1)}\left(t_{n}\right)$ the principal local truncation error at the point $t_{n}$. Hence from our calculations the order of the BSDF is seven and the local truncation error is given as

$$
\left(\frac{2633}{282240}, \frac{187}{26460}, \frac{257}{31360}, \frac{16}{2205}, \frac{1375}{169344}\right)^{T}
$$

where $T$ is transpose.

\subsection{Zero Stability}

The zero stability of the method is concerned with the stability of the difference system in the limit as $h$ tends to zero (see [7]). Thus, as $h \longrightarrow 0$ the difference system (13) tends to

$$
A^{(1)} Y_{\omega}=A^{(0)} Y_{\omega-1} .
$$

Whose first characteristics polynomial $\rho(R)$ given by

$$
\rho(R)=\operatorname{Det}\left[R A^{(1)}-A^{(0)}\right]=R^{4}(1-R)
$$

The block method (13) is zero stable for $\rho(R)=0$ and satisfies $\left|R_{j}\right| \leq 1, j=$ $1, \ldots k$ and for those roots with $\left|R_{j}\right|=1$, the multiplicity does not exceed 1 . Hence the BSDF is zero stable.

\subsection{Consistency and Convergence}

We note that the BSDF is consistent as it has order $p>1$ Since the BSDF (13) is zero stable. According to Henrici [11],

Convergence $=$ zero stability + consistency. Hence the BSDF converges.

\subsection{Linear stability}

The linear stability properties of the BSDF is discussed in the spirit of Hairer and Wanner [10] and determined by expressing it in the form (13) and applying 
the test problem

$$
y^{\prime}=\lambda y, \quad y^{\prime \prime}=\lambda^{2} y, \quad \lambda<0
$$

to yield

$$
Y_{\omega}=Q(z) Y_{\omega-1}, z=\lambda h,
$$

where the matrix $Q(z)$ is given by

$$
Q(z)=\left(A^{(1)}-z B^{(1)}-z^{2} C^{(1)}\right)^{-1} \cdot\left(A^{(0)}+B^{(0)}\right)
$$

The matrix $\mathrm{Q}(\mathrm{z})$ has eigenvalues $\left\{\xi_{1}, \xi_{2}, \ldots, \xi_{5}\right\}=\left\{0,0, \ldots, \xi_{5}\right\}$, where the dominant eigenvalue $\xi_{5}$ is the stability function $\xi(z): \mathbb{C} \rightarrow \mathbb{C}$ which is a rational function with real coefficients given by

$$
\frac{6\left(420+900 z+850 . z^{2}+450 z^{3}+137 z^{4}+20 z^{5}\right)}{2520-7200 . z+9600 z^{2}-7800 z^{3}+4197 z^{4}-1490 z^{5}+300 z^{6}}
$$

The stability domain of the method (or region of absolute stability) $S$ is defined as

$$
S=[z \in \mathbb{C}: R(z) \leq 1]
$$

Specifically, when the left-half complex plane is contained in $S$, the method is said to be A-stable. In Figure 1, the plot with the rectangles representing the zeros and plus signs representing the poles of (19). The plot in white represents the stability region which corresponds to the stability function (19). Clearly, from Figure 1, it is obvious that our method is A- stable since according to Hairer and Wanner [10] there is no pole of the stability function (19) in the left half complex plane.

\section{Computational Aspect of the SBDF}

The scheme is implemented more efficiently as block numerical integrators for equation (5) ) to simultaneously obtain the approximations $\left(y_{n+1}, y_{n+2}, \ldots\right.$, $\left.y_{n+5}\right)^{T}$ without requiring starting values and predictors, $n=0, k, \ldots, N-5$, over sub-intervals $\left[t_{0}, t_{5}\right], \ldots,\left[t_{N-5}, t_{N}\right]$. For example $n=0, \omega=1$ the values for $\left(y_{1}, y_{2}, \ldots, y_{5}\right)^{T}$, are simultaneously obtained over the sub-interval $\left[t_{0}, t_{5}\right]$, as $y_{0}$ is known from (1).

For $n=1, \omega=2,\left(y_{6}, y_{7}, \ldots, y_{10}\right)^{T}$ are simultaneously obtained over the sub-interval $\left[t_{5}, t_{10}\right]$, as $y_{5}$ is known from the previous block. Hence, the subintervals do not over-lap. It should be noted that for linear problems, the code uses Gaussian elimination while the Newton's method is used for nonlinear problems. 


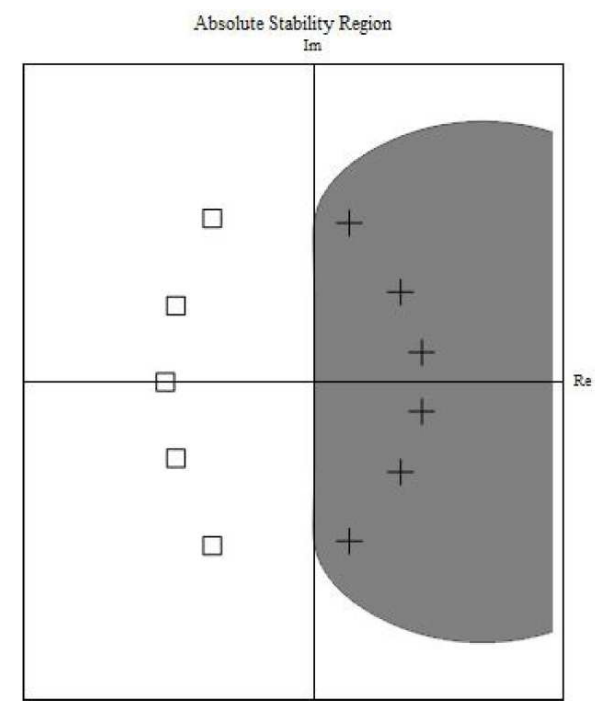

Figure 1: Stability Region

\section{Numerical Examples}

This section deals with some numerical experiments, executed in our written code in MATHEMATICA 8 to illustrate the result derived in the previous sections.

Example 5.1. : Consider the differential-algebraic equations with Hessenberg index-3 given by

$$
\begin{array}{cc}
y_{1}^{\prime}+y_{1}-1=0, & y_{1}(0)=0 . \\
y y_{2}^{\prime}+y_{3}^{\prime}+2 y_{2}-2 y=0, & y_{2}(0)=-1 . \\
y y_{2}=y_{3}-e^{t}, & y_{2}(0)=1 .
\end{array}
$$

The exact solution is given as:

$$
\begin{gathered}
y_{1}(t)=e^{t}-1 \\
y_{2}(t)=2 t-e^{t} \\
y_{3}(t)=(1+t) e^{t}-2 t^{2}
\end{gathered}
$$


Example 5.2. : Our second example is the differential-algebraic equations with Hessenberg index-3 given by

$$
\begin{array}{cc}
y_{1}^{\prime}+y_{1}+y_{2}+t y_{3}-2 t=0, & y_{1}(0)=1 . \\
y_{2}^{\prime}+e^{t} y_{1}+(t+1) y_{2}-t^{2}-t-2=0, & y_{2}(0)=0 . \\
t^{2} y_{2}-t^{3}=0, & y_{3}(0)=1 .
\end{array}
$$

whose exact solution is given as:

$$
\begin{gathered}
y_{1}(t)=e^{-t} \\
y_{2}(t)=t \\
y_{3}(t)=1
\end{gathered}
$$

The results for the two problems are tabulated for $h=0.1$ and compared with the results in $[12]$

Table 1: Exact $y_{1}(t)$ and Numerical solution $y_{1}^{*}(t)$ and $h=0.1$ for Example5.1

\begin{tabular}{|c|c|c|c|}
\hline $\mathrm{t}$ & $y_{1}(t)$ & $y_{1}^{*}(t)$ & $\left|y_{1}(t)-y_{1}^{*}(t)\right|$ \\
\hline 0.0 & 0 & 0 & 0 \\
\hline 0.1 & 0.105170918075 & 0.105170917955 & $1.20 \times 10^{-10}$ \\
\hline 0.2 & 0.221402758160 & 0.221402758069 & $9.10 \times 10^{-11}$ \\
\hline 0.3 & 0.349858807576 & 0.349858807470 & $1.06 \times 10^{-10}$ \\
\hline 0.4 & 0.491824697641 & 0.491824697547 & $9.40 \times 10^{-11}$ \\
\hline 0.5 & 0.648721270700 & 0.648721270595 & $1.05 \times 10^{-10}$ \\
\hline 0.6 & 0.822118800390 & 0.822118800086 & $3.04 \times 10^{-10}$ \\
\hline 0.7 & 1.013752707470 & 1.013752707215 & $2.55 \times 10^{-10}$ \\
\hline 0.8 & 1.225540928492 & 1.225540928212 & $2.80 \times 10^{-10}$ \\
\hline 0.9 & 1.459603111156 & 1.459603110897 & $2.59 \times 10^{-10}$ \\
\hline 1.0 & 1.718281828459 & 1.718281828180 & $2.79 \times 10^{-10}$ \\
\hline
\end{tabular}

Exact $y_{2}(t)=$ Numerical solution $y_{2}^{*}(t)=t$.

Exact $y_{3}(t)=$ Numerical solution $y_{3}^{*}(t)=1$. 
Table 2: comparison of methods for $y_{1}^{*}(t)$ Example5.1

\begin{tabular}{|c|c|c|}
\hline $\mathrm{t}$ & Error in $[12]$ & Error in BSDF \\
0.0 & 0 & 0 \\
\hline 0.1 & $1.00 \times 10^{-10}$ & $1.20 \times 10^{-10}$ \\
\hline 0.2 & $2.00 \times 10^{-10}$ & $9.10 \times 10^{-11}$ \\
\hline 0.3 & $4.00 \times 10^{-10}$ & $1.06 \times 10^{-10}$ \\
\hline 0.4 & $3.00 \times 10^{-10}$ & $9.40 \times 10^{-11}$ \\
\hline 0.5 & $5.00 \times 10^{-10}$ & $1.05 \times 10^{-10}$ \\
\hline 0.6 & $1.30 \times 10^{-9}$ & $3.04 \times 10^{-10}$ \\
\hline 0.7 & $8.00 \times 10^{-9}$ & $2.55 \times 10^{-10}$ \\
\hline 0.8 & $3.10 \times 10^{-8}$ & $2.80 \times 10^{-10}$ \\
\hline 0.9 & $1.05 \times 10^{-7}$ & $2.59 \times 10^{-10}$ \\
\hline 1.0 & $3.01 \times 10^{-7}$ & $2.79 \times 10^{-10}$ \\
\hline
\end{tabular}

Table 3: Exact $y_{2}(t)$ and Numerical solution $y_{2}^{*}(t)$ and $h=0.1$ for Example5.1

\begin{tabular}{|c|c|c|c|}
\hline $\mathrm{t}$ & $y_{2}(t)$ & $y_{2}^{*}(t)$ & $\left|y_{2}(t)-y_{2}^{*}(t)\right|$ \\
\hline 0.0 & -1 & -1 & 0 \\
\hline 0.1 & -0.905170918075 & -0.905170917955 & $1.20 \times 10^{-10}$ \\
\hline 0.2 & -0.821402758160 & -0.821402758069 & $9.10 \times 10^{-11}$ \\
\hline 0.3 & -0.749858807576 & -0.749858807470 & $1.06 \times 10^{-10}$ \\
\hline 0.4 & -0.691824697641 & -0.691824697547 & $9.40 \times 10^{-11}$ \\
\hline 0.5 & -0.648721270700 & -0.648721270595 & $1.05 \times 10^{-10}$ \\
\hline 0.6 & -0.622118800390 & -0.622118800086 & $3.04 \times 10^{-10}$ \\
\hline 0.7 & -0.613752707470 & -0.613752707215 & $2.55 \times 10^{-10}$ \\
\hline 0.8 & -0.625540928492 & -0.6255409282126 & $2.80 \times 10^{-10}$ \\
\hline 0.9 & -0.659603111156 & -0.659603110897 & $2.59 \times 10^{-10}$ \\
\hline 1.0 & -0.718281828459 & -0.718281828180 & $2.79 \times 10^{-10}$ \\
\hline
\end{tabular}

\section{Conclusion}

We have proposed a block second derivative formula of order seven for the solution of differential-algebraic equations DAEs with Hessenberg index-3. The algorithms are self-starting, consistent and provide good accuracy. Numerical examples using the BSDF showed that the method is accurate and efficient for the class of problems being considered.

\section{References}

[1] O.A. Akinfenwa and S.A. Okunuga, Solving Semi-Explicit Index-1 DAE 
Table 4: comparison of methods for $y_{2}^{*}(t)$ Example5.1

\begin{tabular}{|c|c|c|}
\hline $\mathrm{t}$ & Error in [12] & Error in BSDF \\
0.0 & 0 & 0 \\
\hline 0.1 & $1.00 \times 10^{-10}$ & $1.20 \times 10^{-10}$ \\
\hline 0.2 & $2.00 \times 10^{-10}$ & $9.10 \times 10^{-11}$ \\
\hline 0.3 & $4.00 \times 10^{-10}$ & $1.06 \times 10^{-10}$ \\
\hline 0.4 & $3.00 \times 10^{-10}$ & $9.40 \times 10^{-11}$ \\
\hline 0.5 & $5.00 \times 10^{-10}$ & $1.05 \times 10^{-10}$ \\
\hline 0.6 & $1.30 \times 10^{-9}$ & $3.04 \times 10^{-10}$ \\
\hline 0.7 & $7.80 \times 10^{-9}$ & $2.55 \times 10^{-10}$ \\
\hline 0.8 & $3.13 \times 10^{-8}$ & $2.80 \times 10^{-10}$ \\
\hline 0.9 & $1.04 \times 10^{-7}$ & $2.59 \times 10^{-10}$ \\
\hline 1.0 & $3.02 \times 10^{-7}$ & $2.79 \times 10^{-10}$ \\
\hline
\end{tabular}

Table 5: Exact $y_{3}(t)$ and Numerical solution $y_{3}^{*}(t)$ and $h=0.1$ for Example5.1

\begin{tabular}{|c|c|c|c|}
\hline $\mathrm{t}$ & $y_{3}(t)$ & $y_{3}^{*}(t)$ & $\left|y_{3}(t)-y_{3}^{*}(t)\right|$ \\
\hline 0.0 & 1 & 1 & 0 \\
\hline 0.1 & 1.195688009883 & 1.195688008743 & $1.14 \times 10^{-9}$ \\
\hline 0.2 & 1.385683309792 & 1.385683308905 & $8.87 \times 10^{-10}$ \\
\hline 0.3 & 1.574816449848 & 1.574816448801 & $1.05 \times 10^{-9}$ \\
\hline 0.4 & 1.768554576697 & 1.768554575743 & $9.54 \times 10^{-10}$ \\
\hline 0.5 & 1.973081906050 & 1.973081904962 & $1.08 \times 10^{-9}$ \\
\hline 0.6 & 2.19539008062 & 2.195390077522 & $3.10 \times 10^{-9}$ \\
\hline 0.7 & 2.443379602699 & 2.4433796000043 & $2.69 \times 10^{-9}$ \\
\hline 0.8 & 2.725973671286 & 2.725973668275 & $3.01 \times 10^{-9}$ \\
\hline 0.9 & 3.053245911198 & 3.0532459083138 & $2.88 \times 10^{-9}$ \\
\hline 1.0 & 3.436563656918 & 3.436563653765 & $3.15 \times 10^{-9}$ \\
\hline
\end{tabular}

Systems using L-Stable Extended Block Backward Differentiation Formula with Continuous Coefficients Proceedings of the World Congress on Engineering,July 3 - 5,2013, London, U.K. Vol I,(2013), pp. 252 - 256

[2] U. M. Ascher, On symmetric schemes and differential-algebraic equations, SIAM Journal on Scientific Computing, vol. 10, no. 5,(1989), pp. 937 - 949.

[3] K. E. Brenan, S. L. Campbell, and L. R. Petzold, Numerical Solution of Initial Value Problems in Differential-Algebraic Equations, Elsevier, New York, NY, USA, 1989.

[4] M Bayram and E Celik, Chebyshev Approximation for Numerical Solu- 
Table 6: comparison of methods for $y_{3}^{*}(t)$ Example5.1

\begin{tabular}{|c|c|c|}
\hline $\mathrm{t}$ & Error in $[12]$ & Error in BSDF \\
0.0 & 0 & 0 \\
\hline 0.1 & 0.00 & $1.14 \times 10^{-10}$ \\
\hline 0.2 & $1.00 \times 10^{-9}$ & $8.87 \times 10^{-10}$ \\
\hline 0.3 & $1.00 \times 10^{-9}$ & $1.05 \times 10^{-10}$ \\
\hline 0.4 & $1.00 \times 10^{-9}$ & $9.54 \times 10^{-10}$ \\
\hline 0.5 & $3.00 \times 10^{-9}$ & $1.08 \times 10^{-9}$ \\
\hline 0.6 & $1.90 \times 10^{-8}$ & $3.10 \times 10^{-9}$ \\
\hline 0.7 & $9.10 \times 10^{-8}$ & $2.69 \times 10^{-9}$ \\
\hline 0.8 & $3.53 \times 10^{-7}$ & $3.01 \times 10^{-9}$ \\
\hline 0.9 & $1.16 \times 10^{-6}$ & $2.88 \times 10^{-9}$ \\
\hline 1.0 & $3.36 \times 10^{-6}$ & $3.15 \times 10^{-9}$ \\
\hline
\end{tabular}

Table 7: Exact $y_{1}(t)$ and Numerical solution $y_{1}^{*}(t)$ and $h=0.1$ for Example5.2

\begin{tabular}{|c|c|c|c|}
\hline $\mathrm{t}$ & $y_{1}(t)$ & $y_{1}^{*}(t)$ & $\left|y_{1}(t)-y_{1}^{*}(t)\right|$ \\
\hline 0.0 & 1 & 1 & 0 \\
\hline 0.1 & 0.904837418036 & 0.904837417969 & $6.70 \times 10^{-11}$ \\
\hline 0.2 & 0.818730753078 & 0.818730753033 & $4.50 \times 10^{-11}$ \\
\hline 0.3 & 0.740818220682 & 0.740818220632 & $5.01 \times 10^{-11}$ \\
\hline 0.4 & 0.670320046035 & 0.670320045995 & $4.03 \times 10^{-11}$ \\
\hline 0.5 & 0.606530659713 & 0.606530659668 & $4.50 \times 10^{-11}$ \\
\hline 0.6 & 0.548811636094 & 0.548811636011 & $8.30 \times 10^{-11}$ \\
\hline 0.7 & 0.4965853037914 & 0.4965853037222 & $6.92 \times 10^{-11}$ \\
\hline 0.8 & 0.449328964117 & 0.4493289640453 & $7.17 \times 10^{-11}$ \\
\hline 0.9 & 0.406569659741 & 0.406569659675 & $6.60 \times 10^{-11}$ \\
\hline 1.0 & 0.3678794411714 & 0.3678794411026 & $6.88 \times 10^{-11}$ \\
\hline
\end{tabular}

tion of Differential-Algebraic Equation, International Journal of Applied Mathematics \& Statistics, Vol. 2, Issue 1,(2004), pp. 29-36.

[5] E. Celik, E. Karaduman, and M. Bayram, Numerical method to solve chemical differential-algebraic equations, International Journal of Quantum Chemistry, vol. 89,(2002), pp. 447451.

[6] E. Celik and M. Bayram, On the numerical solution of differential-algebraic equations by Pade series, Applied Mathematics and Computation, vol. 137, no. 1,(2003), pp. $151-160$.

[7] S.O. Fatunla, Block methods for second order IVPs, Intern. J. Comput. Math. vol. 41 (1991), pp. 55-63. 
Table 8: comparison of methods for $y_{1}^{*}(t)$ Example5.2

\begin{tabular}{|c|c|c|}
\hline $\mathrm{t}$ & Error in [12] & Error in BSDF \\
0.0 & 0 & 0 \\
\hline 0.1 & $1.00 \times 10^{-10}$ & $6.70 \times 10^{-11}$ \\
\hline 0.2 & $1.00 \times 10^{-10}$ & $4.50 \times 10^{-11}$ \\
\hline 0.3 & $1.00 \times 10^{-10}$ & $5.01 \times 10^{-11}$ \\
\hline 0.4 & $1.00 \times 10^{-10}$ & $4.03 \times 10^{-11}$ \\
\hline 0.5 & $2.00 \times 10^{-10}$ & $4.50 \times 10^{-11}$ \\
\hline 0.6 & $1.60 \times 10^{-9}$ & $8.30 \times 10^{-11}$ \\
\hline 0.7 & $7.20 \times 10^{-9}$ & $6.92 \times 10^{-11}$ \\
\hline 0.8 & $2.76 \times 10^{-8}$ & $7.17 \times 10^{-11}$ \\
\hline 0.9 & $8.87 \times 10^{-8}$ & $6.60 \times 10^{-11}$ \\
\hline 1.0 & $2.52 \times 10^{-7}$ & $6.88 \times 10^{-11}$ \\
\hline
\end{tabular}

[8] C.W. Gear, Numerical Initial Value Problems in Ordinary Differential Equations,Prentice-Hall, Englewood Cliffs NJ. (1971).

[9] C.W. Gear and L. R. Petzold, ODE methods for the solution of differential/algebraic systems, SIAM Journal on Numerical Analysis, vol. 21, no. 4,(1984) pp. $716-728$.

[10] E. Hairer and G. Wanner, Solving Ordinary Differential Equations II, Springer, New York, 1996 .

[11] P. Henrici, Discrete Variable Methods in ODEs, John Wiley, New York, 1962.

[12] M. Karta and E. Celik, On the Numerical Solution of DifferentialAlgebraic Equations with Hessenberg Index-3,Discrete Dynamics in Nature and Society Volume 2012 (2012), Article ID 147240, 12 pages http://dx.doi.org/10.1155/2012/147240

[13] J.D. Lambert, Numerical Methods For Ordinary Differntial Systems, The Initial Value Problem. Wiley, Chichester, New York, (1991).

[14] W. E. Milne, Numerical solution of differential equations, John Wiley and Sons, 1953.

[15] Naghmeh Abasi,Mohamed Suleiman,Zarina Bibi Ibrahim, and Fudziah Ismail, 2-Point and 3-Point BBDF Methods for Solving Semi-Explicit Index1 DAEs Applied Mathematical Sciences, Vol. 6,no. 134, (2012), pp. 6679 6689 
[16] J. D. Rosser, A Runge-Kutta for all seasons, SIAM, Rev.,vol. 9, (1967), pp. $417-452$.

[17] D. Sarafyan Multistep methods for the numerical solution of ordinary differential equations made self-starting, Tech. Report 495, Math. Res. Center, Madison, (1965).

[18] L. F. Shampine and H. A. Watts, Block Implicit One-Step Methods, Math. Comp. 23, (1969), pp. 731 - 740 
